\title{
Orbital ordering and structural distortions in vanadium spinels
}

\author{
Alexander J. Browne ${ }^{1}$, J. Paul Attfield ${ }^{1}$ \\ ${ }^{1}$ Centre For Science At Extreme Conditions, University Of Edinburgh, Edinburgh, United Kingdom \\ E-mail: A.J.Browne@sms.ed.ac.uk
}

\begin{abstract}
Materials with the spinel crystal structure exhibit a diverse range of ground states and properties. These result from the interplay of spin, charge and orbital degrees of freedom over the geometrically frustrated pyrochlore sublattice. In certain spinels the interaction of ordered orbitals induces a structural distortion, due to the formation of clusters of directly bonded atoms called orbital molecules [1]. These clusters influence the properties of the material, thus understanding and manipulating their formation is important both fundamentally and for future functional applications.

Significant examples of orbital molecules include the three atom trimerons formed below the Verwey transition in magnetite [2], and seven atom heptamers found in AIV2O4 [3]. We have recently synthesised GaV2O4, a new orbital molecule material. High-resolution neutron and synchrotron powder diffraction has been used to determine its structure through the ordering temperature and identify the formation of atomic clusters. A full characterisation of the ordering in GaV2O4, relating the structure to the magnetic and physical properties of this material, and a comparison to AIV2O4 will be presented.
\end{abstract}

[1] Attfield, J. P. (2015). APL Mater. 3, 041510

[2] Senn, M. S. et al. (2012). Nature, 481, 173-176

[3] Horibe, Y. et al. (2006). Phys. Rev. Lett. 96, 086406

Keywords: spinel, orbital order, powder diffraction 